\title{
TACKLING THE AFTERGLOW FORWARD-SHOCK MODEL WITH GROND
}

\author{
R. Filgas ${ }^{1,2}$
}

\begin{abstract}
We present the scientific analysis of the GRB afterglow data obtained by the GROND, a seven-channel imager with four optical and three near-infrared detectors. Its unique capability to observe in all bands simultaneously, together with rapid triggering, precise photometry and high temporal resolution, give us the opportunity to study light curves and spectral energy distributions of GRB afterglows in unprecedented detail. This is demonstrated using the observations of three GRB afterglows that are used to put strong constrains on the standard GRB fireball scenario.
\end{abstract}

\section{Introduction}

Even though it is now almost half a century since the Gamma-Ray Bursts (GRBs) were discovered, the exact nature of their emission is still not clearly understood. The leading model for the radiation principle of GRBs is the fireball shock scenario (Mészáros 2002; Meszaros \& Rees 1997; Piran 1999; Zhang \& Mészáros 2004). In this model, the prompt gamma emission is produced by internal shocks when the faster fireball shell catches up with the shell with lower Lorentz factor. The fireball then propagates into the ambient medium. where it produces a blast wave. This external shock is responsible for the long-lived afterglow emission in all wavelengths below gamma-rays.

Most of the afterglow light curves observed before the launch of the Swift satellite (Gehrels et al. 2004) were consistent with this model (Halpern et al. 1999; Stanek et al. 1999). However, the more recent and detailed light curves of afterglows, obtained with the latest generation of GRB instruments capable of high sampling in both time and energy domains, showed features that needed various

\footnotetext{
1 Institute of Experimental and Applied Physics, Czech Technical University in Prague, Horska 3a/22, 12800 Prague 2, Czech Republic; e-mail: robert.filgas@utef.cvut.cz

2 Max-Planck-Institut für extraterrestrische Physik, Giessenbachstraße 1, 85748 Garching, Germany
} 
additions and modifications to the simplest fireball model. This work presents three such cases observed by the Gamma-Ray burst Optical Near-infrared Detector (GROND, Greiner et al. 2008). This instrument has provided high-quality, very well-sampled, simultaneous data in seven bands since 2007, when it was mounted at the $2.2 \mathrm{~m} \mathrm{MPI/ESO} \mathrm{telescope} \mathrm{at} \mathrm{La} \mathrm{Silla} \mathrm{observatory} \mathrm{in} \mathrm{Chile.} \mathrm{The} \mathrm{high-}$ precision data obtained by GROND allow for a detailed study of afterglow light curves (Nardini et al. 2011), jets of GRBs (Krühler et al. 2009), the dust in their host galaxies (Greiner et al. 2011; Krühler et al. 2011a; Schady et al. 2012), their redshifts (Greiner et al. 2009; Krühler et al. 2011b), their associations with SNe (Olivares E. et al. 2012), and provide tools to test the standard fireball scenario and its modifications.

Here we provide details of the GROND and Swift/XRT (in some individual cases together with Swift/UVOT, REM, BOOTES-3 and Stardome) observations of the afterglows of GRB 080413B, GRB 091127 and GRB 091029 and discuss their light curves and spectral energy distributions (SEDs) in the context of the fireball shock model thanks to the very good energy and time-domain coverage of our high-quality data. Throughout the work, we adopt the convention that the flux density of the GRB afterglow can be described as $F_{\nu}(t) \propto t^{-\alpha} \nu^{-\beta}$, where $\alpha$ is the temporal and $\beta$ the spectral index. Unless stated otherwise in the text, all reported errors are at $1 \sigma$ confidence level.

\section{GRB 080413B}

The first of the three presented cases is the afterglow of GRB 080413B (Filgas et al. 2011b), which in the optical/NIR domain shows an initial decay with a slope of $\alpha=0.73 \pm 0.01$, interrupted at roughly $1 \mathrm{ks}$ by a chromatic flattening (Fig. 1a). The decay is later resumed with a steeper temporal slope of $\alpha=0.95 \pm 0.02$ until an achromatic jet break at roughly $330 \mathrm{ks}$. After the jet break the afterglow fades with a steep decay of $\alpha=2.75 \pm 0.16$, which is flattened at the end $\left(>T_{0}+1 \mathrm{Ms}\right)$ by a possible faint host galaxy. The initial decay of the X-ray light curve has the same temporal slope as seen in the optical/NIR wavelengths but the plateau phase is completely missing. The significantly different evolution of the X-ray and optical/NIR light curve can be explained by a two-component jet (see Filgas et al. $2011 b$ and references therein for details).

The initial shallow decay phase in all wavelengths could be the result of the emission of the decelerating narrow jet, which until its jet break dominates the afterglow emission. This jet break is hidden by the onset of the wide jet but from the model we can estimate its time to be at around $3.9 \mathrm{ks}$. From this value we can calculate the opening angle of the narrow jet to be $\theta_{n} \sim 1.7^{\circ}$. Assuming the time of the first $R^{\prime}$ band data point to be upper limit on the time of the emission peak, we calculate the initial Lorentz factor as $\Gamma_{n}>190$. The wide-jet component is in this scenario responsible for the rebrightening of the optical/NIR light curve. The jet break at roughly $330 \mathrm{ks}$ leads to an opening angle of the wide jet of $\theta_{w} \sim 9^{\circ}$ and the initial Lorentz factor, corresponding to the peak of the second jet, is then $\Gamma_{w} \sim 18.5$. The X-ray light curve has a much lower contribution from the second 


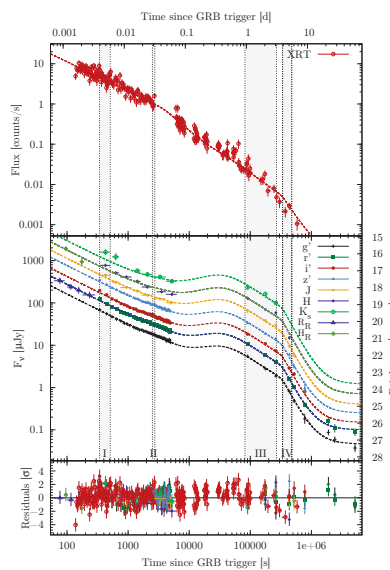

(a) GRB 080413B

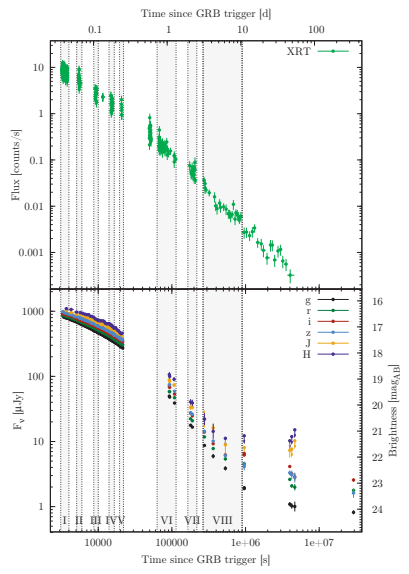

(b) GRB 091127

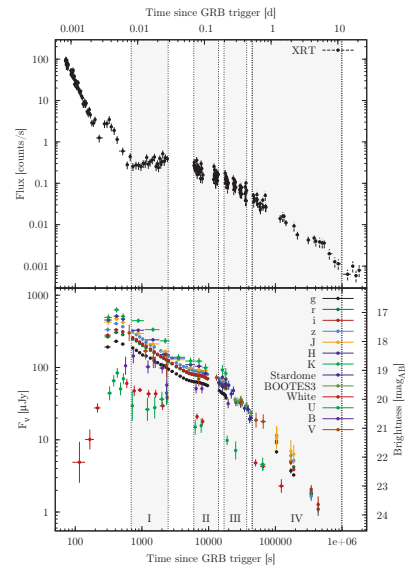

(c) GRB 091029

Fig. 1. Light curves of the X-ray (top panels) and optical/NIR (middle panels) afterglows. Upper limits are not shown for better clarity. Gray regions show the time intervals where the broad-band SEDs are reported.

component and therefore lacks the rebrightening. The case of the afterglow of GRB 080413B shows that we need a structured jet to explain all the features of its optical/NIR light curve and the difference between the X-ray and optical/NIR light curves. Moreover, it shows that GROND can provide observational data good enough to obtain parameters of both components of the jet from the light curve morphology.

\section{$3 \quad$ GRB 091127}

The case of the afterglow of GRB 091127 (Filgas et al. 2011a) proves that what looks like a typical afterglow, can hold the evidence of an extremely rarely observed phenomenon. The X-ray light curve (Fig. 1b) of this afterglow is best fitted using a smoothly broken power-law with an initial decay slope $1.02 \pm 0.04$, a break at around $33 \mathrm{ks}$ and a post-break temporal slope of $1.61 \pm 0.04$. The optical/NIR light curve follows the same shape but with a much flatter initial temporal slope, which further flattens with the increasing wavelength of GROND filters. This suggests a strong color evolution, which is confirmed by measuring the spectral slope of each optical/NIR exposure thanks to simultaneous multi-band observing capabilities of GROND. From this we see that the optical/NIR spectral index rises from $0.23 \pm 0.04$ to $0.80 \pm 0.08$ between 3 and $300 \mathrm{ks}$. In addition, we constructed broad-band optical/NIR to X-ray SEDs at eight different time intervals, indicated in the light curve. Given that these broad-band SEDs proved to be inconsistent with a straight power-law, we used models that include a spectral break between the X-ray and optical/NIR data. This fit shows that the break evolves to larger 
wavelengths in time, through and beyond the GROND bands. However, this fit with the sharp break requires the low-energy spectral index to be time-dependent, contrary to what the theory expects.

To keep the spectral indices constant, we fitted all eight broad-band SEDs simultaneously with two power-laws connected by a smooth break. The fit again shows the break moving towards the lower energies and allows us to obtain not only the speed of the break but also its shape. We identify the spectral break to be the cooling break, one of the frequencies typical for the synchrotron emission of GRB afterglows. The fit-derived smoothness of $2.2 \pm 0.2$ shows that the cooling break is very smooth, as predicted by Granot \& Sari (2002). The calculated speed of the cooling break, which moves with the temporal power-law index of $-1.23 \pm 0.06$, is way faster than theoretical speed of $t^{-0.5}$ (Sari et al. 1998). We explain this by letting the fraction of energy in the magnetic field vary in time. To be consistent with our measurement of the cooling break speed, this microphysical parameter would have to rise in time as $\epsilon_{B} \propto t^{0.49}$.

\section{GRB 091029}

The last presented example is the afterglow of GRB 091029 (Filgas et al. 2012). It's light curve (Fig. 1c) is almost totally decoupled between the X-ray and optical/NIR domains. The X-ray light curve begins with a tail of the GRB emission, interrupted at $\sim 200 \mathrm{~s}$ by an X-ray flare, followed by a shallow rise peaking around $7.4 \pm 1.8 \mathrm{ks}$ and a decay with temporal index of $1.20 \pm 0.04$. On the other hand, the optical/NIR light curve shows a steep $(\alpha=-2.90 \pm 0.67)$ initial rise from the start of the observations until the peak at around $400 \mathrm{~s}$. The decay following the initial peak has a slope of $\alpha=0.58 \pm 0.01$ until around $5 \mathrm{ks}$, when it starts to flatten. Fitting this shallow decay phase between $0.6-5 \mathrm{ks}$ in each optical/NIR band separately shows a steepening of the temporal index with increasing wavelength of the GROND filters, suggesting that the afterglow gets bluer. This is confirmed by measuring the spectral slope $\beta$ of the optical/NIR data as a function of time. This measurement shows that the optical/NIR spectral index decreases from $0.57 \pm 0.04$ to $0.26 \pm 0.03$ between 0.4 and $9 \mathrm{ks}$, and then slowly increases again to a value of $0.49 \pm 0.12$ at around $100 \mathrm{ks}$.

The almost total decoupling of the optical/NIR and the X-ray light curves of the afterglow of GRB 091029 suggests a double outflow origin. This is supported by our finding that the X-ray spectral hardness does not evolve synchronously with the optical spectral hardening at $0.3-10 \mathrm{ks}$. We conclude that the only scenario consistent with all the peculiarities of the observed light curve and spectra is the two-component jet setup, in which the outflows are at different stages of the synchrotron spectral evolution. The flattening of the broad-band SEDs in the optical/NIR region would be a result of the wide jet having both the cooling break $\nu_{c}$ and the injection frequency $\nu_{m}$ between the X-ray and optical/NIR wavelengths, while the narrow jet has only the cooling frequency $\nu_{c}$ between X-ray and optical/NIR bands. As the ratio between these two outflows changes, it would explain the spectral hardening in the optical/NIR bands, while being consistent 
with the X-ray spectral slope staying constant thanks to equal $p$ values in both outflows. The turnover in the spectral evolution at $\sim 10 \mathrm{ks}$ can be explained by the passage of the frequency $\nu_{m}$ through the GROND filters, after which the optical/NIR spectral index would be consistent with the spectral phase of the narrow jet. This scenario is difficult to confirm or disprove, though, by fitting the light curve and SEDs alone because this model has a large number of free parameters.

\section{Conclusions}

The growing number of well-sampled data sets from the latest generation of instruments like the GROND imager once again show that the standard fireball scenario is too simple to explain the observed data for some time now. The simplest fireball model has an increasingly difficult time to explain the complex light curves and spectral evolutions of numerous GRB afterglows. This is demonstrated in this work by three examples, the afterglow of GRB 080413B needed the twocomponent jet to explain its chromatic rebrightening, the afterglow of GRB 091127 needed the energy of the magnetic field to increase in time, and the afterglow of GRB 091029 is very difficult to explain in the fireball shock model framework at all, showing that some major modifications to the fireball scenario or even some alternate models might be needed to cope with our multi-wavelength data.

\section{References}

Filgas, R., Greiner, J., Schady, P., et al., 2012, A\&A, 546, A101

Filgas, R., Greiner, J., Schady, P., et al., 2011a, A\&A, 535, A57

Filgas, R., Krühler, T., Greiner, J., et al., 2011b, A\&A, 526, A113

Gehrels, N., Chincarini, G., Giommi, P., et al., 2004, ApJ, 611, 1005

Granot, J., \& Sari, R., 2002, ApJ, 568, 820

Greiner, J., Bornemann, W., Clemens, C., et al., 2008, PASP, 120, 405

Greiner, J., Krühler, T., Fynbo, J.P.U., et al., 2009, ApJ, 693, 1610

Greiner, J., Krühler, T., Klose, S., et al., 2011, A\&A, 526, A30

Halpern, J.P., Kemp, J., Piran, T., et al., 1999, ApJ, 517, L105

Krühler, T., Greiner, J., Afonso, P., et al., 2009, A\&A, 508, 593

Krühler, T., Greiner, J., Schady, P., et al., 2011a, A\&A, 534, A108

Krühler, T., Schady, P., Greiner, J., et al., 2011b, A\&A, 526, A153

Mészáros, P., 2002, ARA\&A, 40, 137

Meszaros, P., \& Rees, M.J., 1997, ApJ, 476, 232

Nardini, M., Greiner, J., Krühler, T., et al., 2011, A\&A, 531, A39

Olivares, E.F., Greiner, J., Schady, P., et al., 2012, A\&A, 539, A76

Piran, T., 1999, Phys. Rep., 314, 575

Sari, R., Piran, T., \& Narayan, R., 1998, ApJ, 497, L17

Schady, P., Dwelly, T., Page, M.J., et al., 2012, A\&A, 537, A15

Stanek, K.Z., Garnavich, P.M., Kaluzny, J., et al., 1999, ApJ, 522, L39

Zhang, B., \& Mészáros, P., 2004, Int. J. Mod. Phys. A, 19, 2385 
